\title{
Design on Fault Diagnosis Expert System for Railway Signal Equipment
}

\author{
Guangrong Wu \\ Liaoning Railway Vocational and Technical College, Jinzhou, 121000, China
}

352343509@qq.com

Keywords: railway signal equipment; fault diagnosis; expert system; design

\begin{abstract}
In view of the fact that the fault of railway signal equipment is difficult to diagnose, this paper designs an expert system to simulate human experts to make decisions and improve the science of fault diagnosis. The design includes four aspects: First, a system structure composed of a database, a knowledge base, an inference engine, and an interpretation mechanism is designed; secondly, basic data of signal equipment, numerical standards of signal equipment, and equipment failure analysis data are designed. The knowledge base constituted; Thirdly, a functional structure consisting of data preprocessing, fault diagnosis, fault alarm, information prompt, fault statistics and system management is designed. Fourthly, the process of equipment fault diagnosis is designed by means of flow chart. and fourthly, a flowchart was used to design the equipment fault diagnosis process . The successful application of this system will help improve the actual work quality and improve the management and maintenance methods.
\end{abstract}

\section{Introduction}

In the new century, China's railway transportation industry has achieved rapid development. As the main tool for people to travel and transport goods, railway transportation plays an important role. Under the background of increasing speed, it is extremely important to ensure safe operation. Safety is the lifeline of railway transportation and the eternal theme of transport production. It not only affects the production efficiency and economic efficiency of the enterprise itself, but also has a major impact on society and economy. As one of the important equipment of railways, railway signal equipment is directly related to the safety of railway transportation. Under the background of high-speed, high-density, heavy-load and automation development, railway signal equipment plays an increasingly important role in railway transportation. important. Due to factors such as quality and unscientific maintenance, railway signal equipment often fails during operation, which seriously affects the safe operation of the railway system. Fully studying the method of diagnosing and processing the failure of railway signal equipment will help improve the actual work quality and further improve the scientific management and maintenance methods.

There are many types of methods currently used in the fault diagnosis of railway signal equipment. There are three commonly used methods: First, the empirical diagnosis method. With the help of the experience of railway signal equipment maintenance personnel, judge the equipment failure phenomenon and find out the specific location of equipment failure. The disadvantage is that the subjectivity is too strong, but in the actual situation, to a certain extent, it can effectively solve the related fault problem. Second, signal processing diagnostic analysis. The signals sent by the signal equipment are continuously collected, system analysis is performed, and the fault phenomenon of the equipment is judged by comparing the signals. This diagnostic method is widely used in various signal devices, and the operator's experience and professional requirements are not high. The disadvantage is that it is susceptible to environmental and other factors. Third, the neural network method. Neural networks have nonlinear, autonomous learning and parallel computing capabilities. They have strong advantages in fault diagnosis. They can use the existing data in the network to predict faults, and can also be used as pattern recognition algorithms to classify different types of faults. The fault diagnosis system can be established from the perspective of fault knowledge processing. The advantage is that any complex nonlinear relationship can be mapped, 
and the learning rules are simple. The disadvantage is that everything is inferred into numerical calculations and the result is bound to lose information.

Artificial intelligence and expert system are the development direction of fault diagnosis of railway signal equipment in the future. Artificial intelligence is the simulation of human thinking logic, through complex series of thinking, solving problems that need to be diagnosed, and showing significant advantages in practice. The expert system is the input of domain experts' knowledge and experience in the system, comprehensive use of multiple diagnostic techniques for reasoning, and expert analysis of problems. An expert system is a computer program that can handle faults at the level of experts in certain areas. Due to the integration of many expert knowledge, in some aspects even more than human experts. The railway signal fault diagnosis expert system is a program system with a large amount of specialized knowledge. Artificial intelligence technology is applied to reasoning based on domain knowledge provided by a number of experts to simulate human experts to make decisions and solve problems that require experts to solve. The scientific combination of expert system and signal equipment fault diagnosis knowledge, the use of various reasoning techniques, and the full use of their respective expertise will increase the intelligence level of the diagnostic system and make it an important means for fault diagnosis of railway signal equipment.

\section{Structure on Fault Diagnosis Expert System for Railway Signal Equipment}

diagnosis expert system is based on the data of the signal monitoring system and consists of three parts: data preprocessing, fault diagnosis, and generation and maintenance. Among them, the fault diagnosis consists of man-machine interface, inference engine, knowledge base, database, knowledge base maintenance and interpretation mechanism, etc. The system structure is shown in Fig. 1.

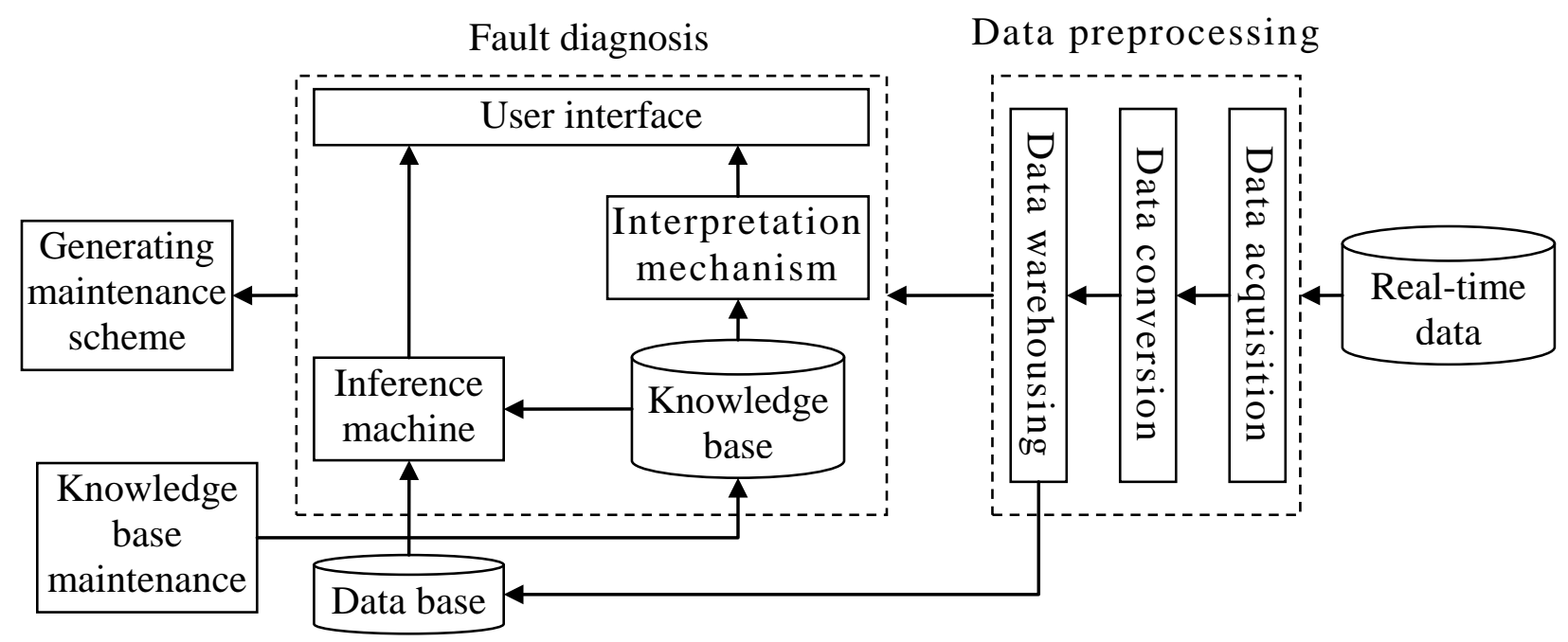

Fig.1. Structure on fault diagnosis expert system for railway signal equipment

The important components shown in Fig. 1 are briefly described as follows:

(1) Database Data base. A well-structured database system is the basis for constructing an expert system. It is used to store all the information generated during the operation of the system, as well as the required raw data, including user input information, inference intermediate results, and inference process records. The state of the database consisting of various facts, propositions, and relationships is both the basis for the inference engine's choice of knowledge and the source of the reasoning mechanism for the reasoning mechanism.

(2) Knowledge Base. The knowledge base is the connection between entities and entities used to store knowledge, and is divided into three categories: rules, facts, and meta-knowledge. It mainly includes definitions of various terms, language descriptions, corresponding consistency, integrity constraints, and meta-rules for reasoning control. Part of the meta-knowledge is stored in the 
concept table. The facts and rules are stored in the fact table and the rules table respectively. The meta-knowledge is also stored in text form.

(3) Inference machine. The inference engine simulates the thinking process of domain experts and controls and executes the solution to the problems. It is a mechanism that identifies, selects, and matches the rules in the knowledge base and obtains the results based on the information of the current problem. In the reasoning process, a series of inference rules expressed in a certain language are interpreted and executed, and relevant knowledge is selected from the knowledge base according to the inference strategy, and the evidence provided by the user is matched until a conclusion is reached.

(4) Interpretative mechanism. The interpretive mechanism needs to help users understand the static knowledge and dynamic reasoning process of the system at any time. The interpretation mechanism consists of a set of programs to track and record the reasoning process. When the user asks for an explanation, it will do the corresponding processing according to the requirements of the problem. The explanation mechanism should provide a good human-computer interaction interface, which is easy for the user to operate. The explanation information can be sent to the screen, document or other output device in a timely manner in a form acceptable to the user.

\section{Knowledge Base on Fault Diagnosis Expert System for Railway Signal Equipment}

Knowledge management is the organization, management, and maintenance of knowledge. Specifically include knowledge classification, knowledge organization and storage, knowledge retrieval, knowledge addition, knowledge deletion, knowledge modification, knowledge copying and dumping, and knowledge consistency, integrity, and redundancy check. The structure of the knowledge base system is shown in Fig. 2. The functional modules include knowledge base establishment, knowledge base update (including add, delete, change), knowledge acquisition and knowledge query. The user interface is a means to provide users with the use of knowledge (inquiry, addition, deletion, change, etc.).

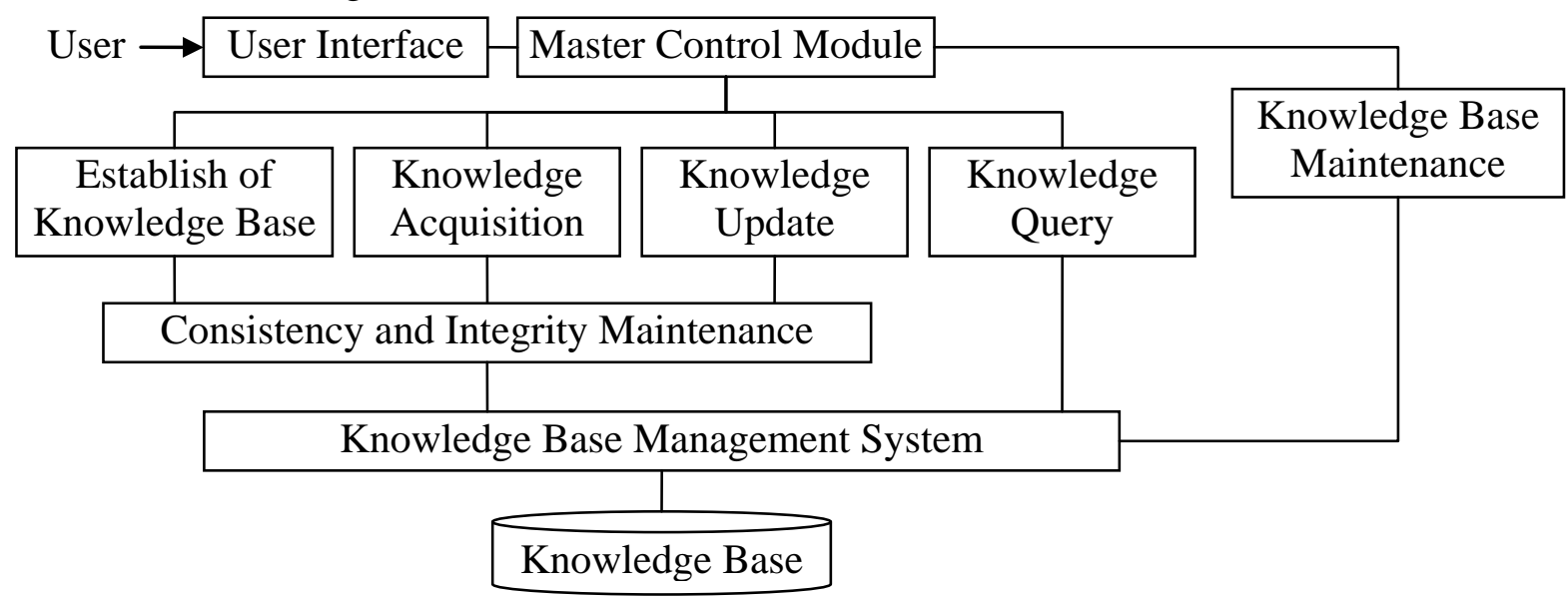

Fig.2. System structure of knowledge base

(1) Signal equipment basic data knowledge base. According to the functional principle of each device related to the signal device, it is classified and classified, and includes the standard circuit diagram of the signal device. Including: basic data of relays, basic data of power supply equipment, basic data of signal generators and signal indicators, basic data of switching and locking equipment, basic data of track circuits, basic data of station interlocking systems, basic data of interval blocking equipment, and crossing equipment Fundamental data, lightning protection basic data of signal equipment, etc., and will be revised along with the updating and upgrading of signal equipment.

(2) Numerical standard knowledge base of signal equipment. For the basic data encoding of signal equipment, the technical standards of all signal equipment are classified and summarized to ensure the comparability of the real-time monitoring data of the signal equipment, beyond the standard range, and the alarm prompts are raised, and the reasons for exceeding the standards are 
analyzed against the knowledge base. Including: Microcomputer monitoring data format standard knowledge base, signal analog analysis standard knowledge base, signal control system logic timing standard knowledge base and so on.

(3) Equipment failure analysis data knowledge base. A total of 14 types of failure analysis knowledge bases are included: Console buttons indicate lamp failures, signal lighting circuit failures, signal repeater failures, track circuit failures, switch failures, normal unlocking failures, cancellation of access failures, manual unlocking failures , Fault unlock circuit fault, guide path lock fault, boot unlock fault, boot total lock fault, circuit fault in the area blocking device, power supply device fault.

\section{Function on Fault Diagnosis Expert System for Railway Signal Equipment}

Functional design is represented by a functional structure model used to describe the hierarchical structure of software functions. A rectangular box represents a module in the structure diagram. The connection line between the rectangles represents the call relationship. The rectangle box on the upper layer represents the module, and the module represented by the lower rectangle box is called. Modular design should follow the principles of abstraction, gradual refinement, modularity, and information hiding. Modular design of software can have different levels of abstraction. At the highest level of abstraction, the language description of the environment in which the problem is located can be used. At the lower level of abstraction, procedural methods are used; stepwise refinement is a top-down design strategy, and the software architecture is structured in a top-down manner, with process details and data at all levels. The details are gradually refined until they can be implemented in the language of the programming language. The functional design results of this system are shown in Fig. 3.

\begin{tabular}{|c|c|}
\hline \multicolumn{2}{|c|}{ Design on Fault Diagnosis Expert System for Railway Signal Equipment } \\
\hline Data preprocessing & Information hints \\
\hline Data acquisition & Section information \\
\hline Data conversion & Switch information \\
\hline Data analysis & Track information \\
\hline Data warehousing & Signal machine \\
\hline Fault diagnosis & Fault statistics \\
\hline Fault level determination & According to time statistics \\
\hline Fault diagnosis & According to equipment statistics \\
\hline Knowledge base maintenance & According to line statistics \\
\hline \begin{tabular}{|c|} 
Database maintenance \\
\end{tabular} & According to region statistics \\
\hline Fault alarm & System management \\
\hline Section fault alarm & User authorization \\
\hline Switch fault alarm & Data backup \\
\hline Track fault alarm & Data recovery \\
\hline Signal machine fault alarm & Configuration management \\
\hline
\end{tabular}

Fig. 3. Functional framework on fault diagnosis expert system for railway signal equipment 


\section{Fault Diagnosis Process on Fault Diagnosis Expert System for Railway Signal Equipment}

The fault diagnosis of railway signal equipment is the core function of the expert system. The diagnosis process is shown in Fig. 4.

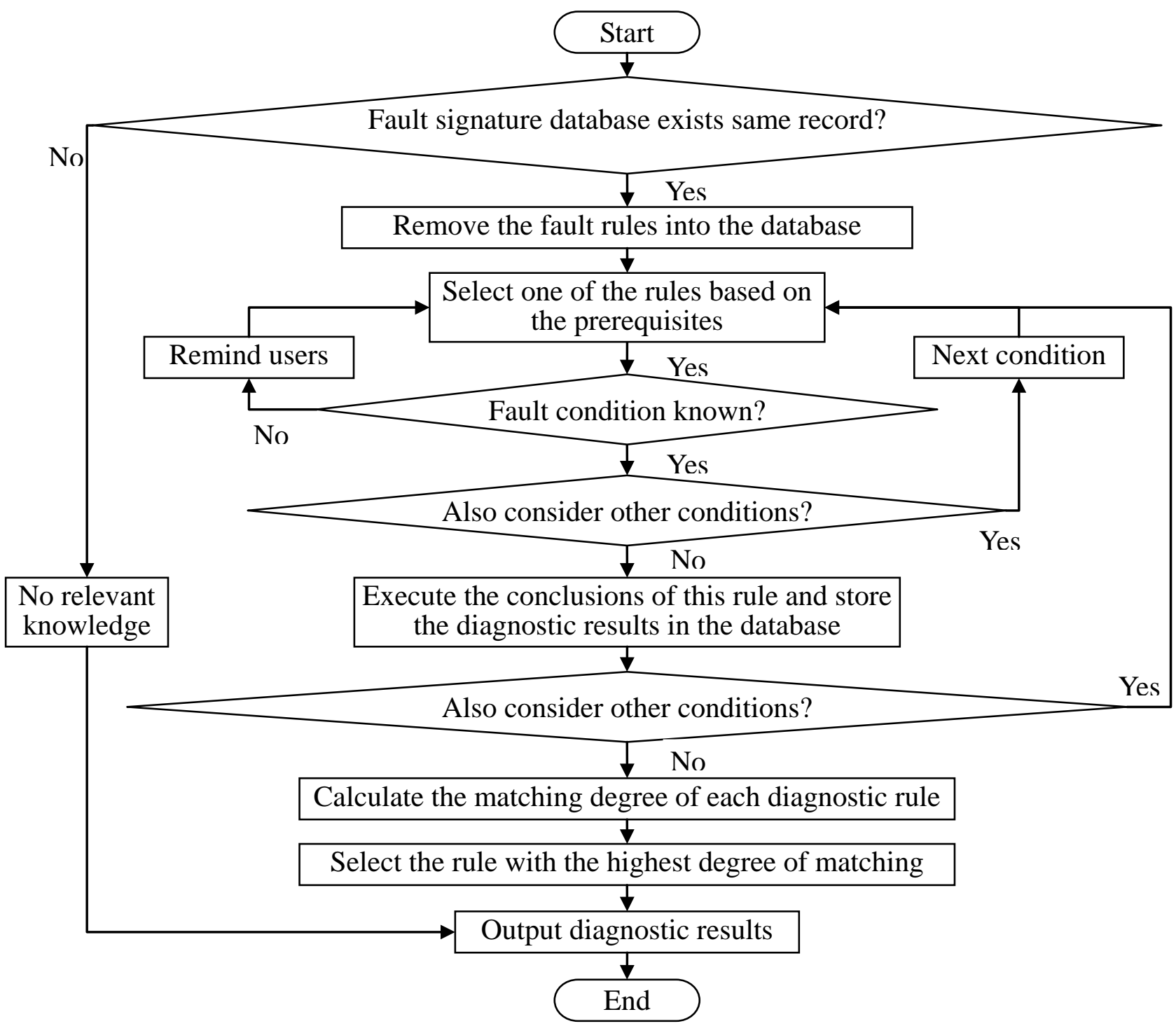

Fig. 4. Fault diagnosis process on fault diagnosis expert system for railway signal equipment

The fault diagnosis of railway signal equipment shown in Fig. 4 is briefly described as follows:

(1) Ask questions and apply expert systems for analysis;

(2) Compare the faults that have occurred with the fault features that have been stored in the system and check whether the same records exist;

(3) If the same fault record is found in the system library, the rule will be activated;

(4) After all the rules of the same record are activated, it is analyzed whether each condition of the rule is the same as the current failure. If the condition of a rule is unknown, ask questions and get more information about the fault through the user's answers;

(5) If the conditions of the rule are all consistent with the conditions of the failure, the execution result of this rule will be stored;

(6) Check if there are other activated rules, if any, execute the loop;

(7) Calculate the credibility of all activation rules;

(8) Use the most reliable troubleshooting method as a maintenance plan;

(9) If there is no corresponding rule, the output "No relevant knowledge", the user will again look for other information. 


\section{References}

[1] B. Y. Zhang, C. H. Liang, Y. Y. Li, "Research on Fault Diagnosis Expert System for Railway Signal Equipment," Railway Signalling \& Communication, vol. 46, no. 9, pp. 26-28, 2010.

[2] L. Y. Wang, "Railway Signal Equipment Fault Diagnosis Base on Expert System," Master's degree of Jinlin University, 2015.

[3] F. Q. Liu, "Analysis of fault diagnosis method for railway signal equipment," http://www.zhazhi.com/lunwen/gyjs/gzzdlw/127607.html, 2018-5-10.

[4] B. Y. Gu, "Analysis of common problems in fault diagnosis of railway signal equipment," http://www.chinaqking.com/yc/2016/619336.html, 2018-5-10.

[5] J. Tan, Y. P. Zhang, "Research on Knowledge Base of Fault Diagnosis Expert System for Railway Signal Equipment," Railway Operation Technology, vol. 17, no. 1, pp. 1-3, 2011.

[6] Q. Q. Zhang, "Design Research on Fault Diagnosis Expert System for Railway Signal Equipment," Technological Development of Enterprise, vol. 35, no. 9, pp. 28-29, 2016. 